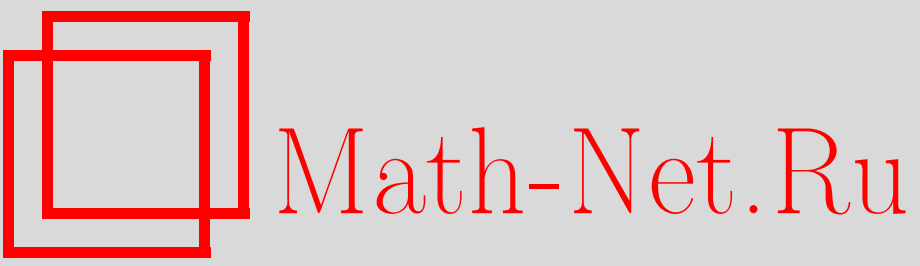

О. В. Мантуров, Игровой подход к ценообразованию в условиях конкуренции, УМН, 1996, том 51, выпуск 1, 155-156

DOI: https://doi.org/10.4213/rm930

Использование Общероссийского математического портала Math-Net.Ru подразумевает, что вы прочитали и согласны с пользовательским соглашением

http://www.mathnet.ru/rus/agreement

Параметры загрузки:

IP: 54.92 .164 .108

26 апреля 2023 г., 18:11:43 


\title{
ИГРОВОЙ ПОДХОД К ЦЕНООБРАЗОВАНИЮ В УСЛОВИЯХ КОНКУРЕНЦИИ
}

\author{
О.В. МАнтуров
}

Пусть две конкурирующие стороны - коммерсант один и коммерсант два - находятся в следуюших условиях. Им известно, что некий продавец согласен продать товар за $a$ рублей, а некоторьй покупатель согласен заплатить за этот товар $b$ рублей, $a<b$. Как первый, так и второй коммерсант желают получить прибыль от разницы между ценой покупки товара у продавца и ценой продажи товара покупателю.

Правила покупки товара таковы: каждый из коммерсантов независимо от конкурента сообщает продавцу цену, которую он согласен заплатить (эта информация недоступна конкурирующей стороне). Продавец продает товар тому коммерсанту, который предложил более высокую цену, а в случае совпадения цен каждый коммерсант покупает половину товара, предназначенного к продаже.

Таким образом, победа на торгах приносит победителю доход в $b-a$ рублей; в случае, когда оба коммерсанта предложили одинаковые суммы, прибыль каждого коммерсанта составит $(b-a) / 2$ рублей.

Как в теории, так и на практике невозможно допускать в качестве цен произвольные вещественные числа между $a$ и $b$. Условимся, что в распоряжении коммерсантов имеются следующие возможности для участия в торгах: каждый из коммерсантов может назвать в качестве цены одно из чисел

$$
a, a+\frac{(b-a)}{n}, a+\frac{2(b-a)}{n}, \ldots, a+\frac{n(b-a)}{n},
$$

где $n$ - некоторое заданное натуральное число.

Пусть стратегия первого коммерсанта состоит в том, что указанные выше цены он называет с вероятностями

$$
p_{0}, p_{1}, p_{2}, \ldots, p_{n}
$$

соответственно, а стратегия второго коммерсанта определяется вероятностями

$$
q_{0}, q_{1}, q_{2}, \ldots, q_{n}
$$

Упорядоченные наборы чисел (2) и (3) будем обозначать через $\vec{p}$ и $\vec{q}$, соответственно. Будем считать, что $a=0, b=n$, и числа (1) имеют вид $0,1,2, \ldots, n$. Это не внесет серьезных изменений в нижеследующую постановку задачи.

Имеем две матрицы $F$ и $G$ размера $n \times n$ с элементами

$$
f_{i j}=\left\{\begin{array}{lll}
0 & \text { при } i<j, \\
(b-a) / 2 & \text { при } i=j, \\
n-i & \text { при } j<i,
\end{array}\right.
$$

$i=0,1,2, \ldots, n-1$,

$$
g_{i j}= \begin{cases}0 & \text { при } j<i, \\ (b-a) / 2 & \text { при } i=j, \\ n-i & \text { при } i<j,\end{cases}
$$

$i=0,1,2, \ldots, n-1$. Элементы $f_{i j}, g_{i j}$ матриц $F, G$ означают выигрыши коммерсантов при назначении цен: $i$ рублей первым из них и $j$ рублей вторым.

При описанных выше условиях следующее поведение конкурирующих коммерсантов является в известной мере разумным и позволяет поставить точную математическую задачу об оптимальных стратегиях и справедливой цене. Средний выигрыш в этой игре однозначно определяет среднюю цену, по которой будет куплен товар у продавца. Эта цена будет, очевидно, не ниже $a$ и не выше $b$. Ее величина будет отражать влияние конкуренции. 
Постановка задачи и ее численное решение для небольших значений $n$ является основной целюю данной работы.

При фиксированном наборе $\overrightarrow{p^{*}}=\left(p_{0}^{*}, p_{1}^{*}, p_{2}^{*}, \ldots, p_{n}^{*}\right)$ математическое ожидание вьпирыша второго коммерсанта $g=\sum_{i=0}^{n-1} g_{i j} p_{i}^{*} q_{j}$. Естественно, второй коммерсант, заботясь о максимальном своем выигрыше, должен выбрать стратегию $q^{*}$ такую, что $\sum_{i j} g_{i j} p_{i}^{*} q_{j}^{*}>\sum_{i j} g_{i j} p_{i}^{*} q_{j}$ для произвольного $q^{*}=\left(q_{0}, q_{1}, q_{2}, \ldots, q_{n}\right), q_{j} \geqslant 0, j=0,1,2, \ldots, n, \sum_{j=0}^{n} q_{j}=1$.

Линейная функция $g$ имеет вид $g=\sum_{j=1}^{n-1} A_{j} q_{j}$, где $A_{j}=\sum_{i=0}^{n-1} g_{i j} p_{i}^{*}$.

Очевидно, что если среди коэффициентов $A_{j}$ имеется единственный максимальный (пусть для определенности $\left.A_{k}>A_{j}, j=0,1,2, \ldots, k-1, k+1, \ldots, n-1\right)$, то максимальное значение функции $g\left(\overrightarrow{p^{*}}, \vec{q}\right)$ достигается при $\overrightarrow{q^{*}}=(0,0, \ldots, 0,1,0, \ldots, 0)$ (единица находится на $k$-м месте) и равно $\sum_{i=0}^{n-1} g_{i j} p_{i}^{*}$. Разумеется, это значение зависит от изначально взятых $p_{0}, p_{1}, p_{2}, \ldots, p_{n}$. Выберем эти вероятности так, чтобы функция $f\left(\vec{p}, \overrightarrow{q^{*}}\right)$ при установленном значении $q^{*}$ достигала максимального значения.

Обе описанные выше задачи (нахождения максимума $g$ по $\vec{q}$ при фиксированном $\overrightarrow{p *}$ и нахождения максимума $f$ по $\vec{p}$ при найденном значении $\vec{q}^{*}$ ) являются задачами линейного программирования.

Ниже дается точная математическая постановка этих задач и приводится их численное решение для $n=4, \ldots, 10$.

Напомним, что коэффициенты $A_{j}$ в разложении функции $g$ по $q_{0}, q_{1}, q_{2}, \ldots, q_{n}$ при фиксированных $p_{0}, p_{1}, p_{2}, \ldots, p_{n}\left(g=\sum_{i=0}^{n-1} A_{i j} q_{j}\right)$ имеют вид: $A_{j}=\sum_{i=0}^{n-1} g_{i j} p_{i}$ и являются линейными функциями от $p_{0}, p_{1}, p_{2}, \ldots, p_{n-1}$. Легко видеть, что при выполнении условий:

$$
A_{0}=A_{k}<=0, A_{1}=A_{k}<=0, A_{2}=A_{k}<=0, \ldots, A_{n-1}=A_{k}<=0,
$$

$\sum_{i=0}^{n} q_{i}=1$, максимальное значение линейной функции $g$ от переменных $q_{0}, q_{1}, q_{2}, \ldots, q_{n-1}$ достигается при $q_{k}=1$ и $q_{i}=0, i=0,1,2, \ldots, k-1, k+1, \ldots, n-1$, и равно $A_{k}\left(p_{0}, p_{1}, p_{2}, \ldots, p_{n-1}\right)$. Рассмотрим следующую задачу линейного программирования: найти максимум линейной функции $A_{k}\left(p_{0}, p_{1}, p_{2}, \ldots, p_{n-1}\right)$ при условии, что переменные $p_{0}, p_{1}, p_{2}, \ldots, p_{n-1}$ неотрицательны и удовлетворяют условиям (4). В силу принятых выше соглашений и обозначений поставленная задача полностью определена натуральными числами $n$, $k \leqslant n$. Обозначим эту задачу через $\operatorname{Prob}(n, k)$.

Для численного решения задач $\operatorname{Prob}(n, k)$ был применен стандартный алгоритм симплексного метода. Ниже приводятся результаты соответствующих компьютерных вычислений. Через $U$ и $V$ обозначены средние вьпгрыши первого и второго коммерсанта, соответственно, $Z$ означает среднюю цену, по которой куплен товар (при условии, что коммерсанты торговались следуя указанным выше принципам), $Y$ означает отношение $Z$ к $b-a$ :

$\operatorname{Prob}(4,1) ; \vec{p}=(1 / 2,0,1 / 2,0,0) ; U=1, V=2, Z=1, Y=1 / 4=0.25 ;$

$\operatorname{Prob}(5,1) ; \vec{p}=(1 / 4,1 / 4,0,1 / 2,0,0) ; U=3 / 2, V=7 / 4, Z=7 / 4, Y=7 / 20=0.35 ;$

$\operatorname{Prob}(6,1) ; \vec{p}=(9 / 35,6 / 35,10 / 35,10 / 35,0,0) ; U=65 / 35, V=69 / 35, Z=76 / 35, Y=$ $76 / 210=0.361905$

$\operatorname{Prob}(7,1) ; \vec{p}=(4 / 11,2 / 11,0,3 / 11,2 / 11,0,0,0,0) ; U=24 / 11, V=34 / 11, Z=19 / 11$, $Y=19 / 77=0.246753$

$\operatorname{Prob}(8,1) ; \vec{p}=(25 / 84,0,10 / 84,14 / 84,7 / 84,28 / 84,0,0,0,0) ; U=31 / 12, V=235 / 84, Z=$ $220 / 84, Y=220 / 672=0.327381$;

$\operatorname{Prob}(9,1) ; \vec{p}=(45 / 158,15 / 158,0,20 / 158,8 / 158,34 / 158,36 / 158,0,0,0,0) ; U=464 / 158$, $V=465 / 158, Z=493 / 158, Y=493 / 1492=0.346695$;

$\operatorname{Prob}(10,1) ; \quad \vec{p}=(245 / 687,70 / 687,0,90 / 687,30 / 687,138 / 687,114 / 687,0,0,0,0,0) ; \quad U=$ $2271 / 687, V=2765 / 687, Z=1834 / 687, Y=1834 / 6870=0.266958$.

Принято редколлегией 30.10 .1995 